\title{
The 370-Da Inhibitor of the Sodium Pump in the Plasma of Haemodialysis Patients
}

\author{
David Makanjuolaa,b Gwyn A. Lord ${ }^{c, d}$ Philip J. Hilton ${ }^{e}$

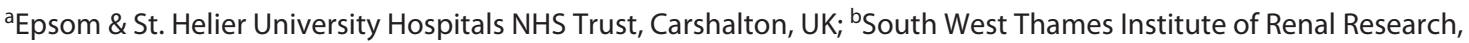 \\ St. Helier Hospital, Carshalton, UK; ' Toxicology Department, Public Health England, Centre for Radiation, Chemical

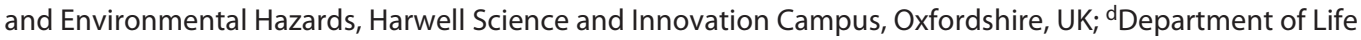 \\ Sciences, University of Suffolk, Ipswich, UK; 'St Thomas' Hospital, London, UK
}

\section{Keywords}

Renal failure · Haemodialysis - Sodium pump · Hypertension . Liquid chromatography/mass spectrometry

\begin{abstract}
Background: Previous studies have shown that a molecule of mass $370 \mathrm{Da}$ that inhibits the sodium pump can be extracted from human placentas and from the concentrated plasma or ultrafiltrate of volume-expanded patients. Aim: This study aimed to study the abundance of the 370-Da molecule and its changes across dialysis in a population of patients with renal failure treated by haemodialysis. Methods: Four millilitres of pre- and post-dialysis blood samples $(2 \mathrm{~mL}$ plasma) were taken from patients receiving intermittent haemodialysis and analysed by high-performance liquid chromatography coupled to high sensitivity mass spectrometry. Results: In over half of the study population, the 370-Da molecule was present in abundance that exceeded the limit of quantitation. Most patients experienced a marked fall in the abundance of the molecule over a haemodiafiltration session, though exceptions were seen in 2 individuals, both of whom showed clear evidence for the presence of 2 structural isomers of the 370-Da molecule. Conclusions: Advanced renal failure is frequently accompanied by an increased abundance of a 370-Da inhibitor of the sodium pump and that abundance is strongly impacted by haemo-
\end{abstract}

karger@karger.com www.karger.com/kbr

Karger"
(C) 2021 The Author(s)

Published by S. Karger AG, Basel

This is an Open Access article licensed under the Creative Commons Attribution-NonCommercial-4.0 International License (CC BY-NC) (http://www.karger.com/Services/OpenAccessLicense), applicable to the online version of the article only. Usage and distribution for commercial purposes requires written permission. dialysis. The technique described here could readily be applied to other clinical situations where sodium pump inhibition might be anticipated, such as hypertension, pregnancy, and foetal medicine, and thereby lead to a better understanding of the physiology and pathophysiology of these conditions.

(C) 2021 The Author(s)

Published by S. Karger AG, Basel

\section{Introduction}

The sodium pump is an electrogenic transmembrane system situated on the outer (plasma) membrane of the cell and is known to be an $\mathrm{Na}^{+} / \mathrm{K}^{+}$ATPase [1]. The concept that the sodium pump of humans is endogenously regulated rather than simply responding to intra- and extracellular ionic concentrations arose from several observations. These include one in which volume-expanded animals develop a factor in their plasma, distinct from the natriuretic peptides, that inhibits the sodium pump (termed the natriuretic hormone) [2], that the plasma of uraemic individuals has similar properties [3], and that patients with untreated hypertension show inhibition of the cellular sodium pump of leucocytes [4], with this effect being transmissible to normal cells by incubation in their plasma [5]. A number of candidates for this role have been proposed. A molecule indistinguishable from 
the plant poison, ouabain (a multiply hydroxylated cardiac glycoside of mass $584 \mathrm{Da}$ with the molecular formula $\mathrm{C}_{29} \mathrm{H}_{44} \mathrm{O}_{12}$ ), was observed in a concentrated extract of a large volume of plasma obtained at therapeutic plasma exchange [6]. Another with the characteristics of the toad venom marinobufagenin (IUPAC name marinobufagin) - a bufadienolide of mass $400 \mathrm{Da}$ with the molecular formula $\mathrm{C}_{24} \mathrm{H}_{32} \mathrm{O}_{5}$ - has also been reported [7]. In our hands, a molecule of mass $370 \mathrm{Da}$ characterized by mass spectrometry (MS) was repeatedly demonstrated in inhibitory fractions of placental extract [8] and the plasma of volume-expanded individuals $[9,10]$. Accurate mass determination made it possible to assign it a molecular formula of $\mathrm{C}_{24} \mathrm{H}_{34} \mathrm{O}_{3}$. The protonated adduct of this molecule (the only ion species seen in MS on the Orbitrap mass spectrometer used in this current study) had a calculated exact mass $(\mathrm{m} / \mathrm{z})$ of 371.2581 . We believe that this molecule is a realistic candidate for the chemical equivalent of the biological "natriuretic hormone."

A major obstacle to progress in this area of research however has previously been the requirement for very large amounts of source material (litres or kilograms) in order to obtain detectable quantities of this inhibitor of the sodium pump. We report here studies to examine whether the 370-Da sodium pump inhibitor can be detected by high-sensitivity MS in a blood sample of small enough size (a $4-\mathrm{mL}$ vacutainer) to be clinically relevant in patients receiving intermittent haemodialysis.

\section{Mass Spectrometry}

MS is recognized as being the most useful and informative analytical technique for partial structural elucidation of biomolecules. Unequivocal determination of structure relies on additional support from other spectroscopic techniques, such as nuclear magnetic resonance spectroscopy, or X-ray crystallography [11]. It is often the case that large amounts of the compound are not easily obtained, and MS provides extremely useful initial information as it is able to gain structural information on molecules at nano- or picomolar levels, which is orders-ofmagnitude below these other techniques.

Analysis by MS requires ionisation of the molecule, and this is achieved by a variety of techniques, depending on the nature of the compound, but electrospray ionisation (ESI) is the predominant technique for biomolecules [12]. This method lends itself to coupling with liquid chromatography (LC) for the prior separation of complex mixtures, resulting in the powerful, synergistic technique of LC/MS. ESI inherently involves addition of adducts to the molecule $(\mathrm{M})$, such as $\mathrm{H}^{+}$or $\mathrm{Na}^{+}$when used in the positive mode, which is most often the case, forming [M $+\mathrm{H}]^{+}$and $[\mathrm{M}+\mathrm{Na}]^{+}$ions, respectively. Molecular composition and chemical formula may be obtained using a high-resolution mass spectrometer, and the calculated "exact mass" may be compared with the experimentally determined "accurate mass" [13].

In our previous work, we used fast atom bombardment and later ESI, coupled principally with double quadrupole analysers, the principal limitation of which was comparatively low sensitivity. We have now been able to use the high sensitivity of the Orbitrap mass spectrometer coupled with its ability to provide the accurate mass of each ion it detects to 4 decimal places with an error invariably $<2 \mathrm{ppm}$ and usually $<1 \mathrm{ppm}$. The advantage of this is that in addition to knowing the molecule's chromatographic retention time, its molecular formula is unequivocally provided.

\section{Methods}

Blood samples of dialysis patients were obtained from the "arterial" end of the dialysis circuit into Becton-Dickinson lithium heparin vacutainers with a whole blood volume of $4 \mathrm{~mL}$. Control samples were taken from 11 self-declared healthy volunteers. The clinical measurements undertaken in the controls were an assessment of the blood pressure, taken at rest, by automated sphygmomanometry, and a biochemical screen.

Twenty-two patients receiving almost invariably 3 times weekly haemodiafiltration (HDF) were studied. Dialysis was normally performed for 3.5-4 h using either Fresenius 5008 or Nikkiso dialysis machines. The dialysers used were polysulfone membranes of the Fresenius FX range (FX60, FX80, or FX100), with HDF replacement volumes of $\sim 15 \mathrm{~L}$.

Samples were prepared by spinning at 3,000 rpm for $10 \mathrm{~min}$, the resulting plasma being maintained at $4^{\circ} \mathrm{C}$ prior to analysis. Solidphase extraction (SPE) was performed with octadecyl (C18)-bonded silica SPE cartridges (Sep-Pak) that had been preconditioned with $5 \%$ ( $\mathrm{vol} / \mathrm{vol}$ ) acetonitrile in water. The retained solutes were washed with the same solution and then eluted in $100 \%$ acetonitrile. The solutions were then taken to dryness using a vacuum centrifuge at $35^{\circ} \mathrm{C}$ and resuspended in $100 \mu \mathrm{L} \mathrm{50 \%}$ acetonitrile/50\% water ( $\mathrm{vol} / \mathrm{vol}$ ) before centrifuging and taking the supernatant for analysis.

\section{Liquid Chromatography/Mass Spectrometry}

Ultra-high-performance liquid chromatography was carried out on a Thermo Scientific (Thermo Fisher Scientific, Hemel Hempstead, UK) UltiMate 3000 UPLC system using a Thermo Scientific reversed phase column (Accucore C18 $100 \times 2.1 \mathrm{~mm} 2.6$ $\mu \mathrm{m})$ at $35^{\circ} \mathrm{C}$ with a flow rate of $0.3 \mathrm{~mL} / \mathrm{min}$ and injecting $10 \mu \mathrm{L}$ of sample via the autosampler. A linear gradient elution was used from $100 \%$ solvent A (95\% water, $5 \%$ acetonitrile with added $0.1 \%$ formic acid vol $/ \mathrm{vol} / \mathrm{vol}$ ) to $100 \%$ solvent $\mathrm{B}$ (acetonitrile with added $0.1 \%$ formic acid vol/vol) over $35 \mathrm{~min}$, following an initial 2-min hold at $100 \%$ solvent A. The column eluent was coupled to a highresolution mass spectrometer (Thermo Orbitrap Q Exactive Plus with ESI), operating in positive mode $m / z 100-1,000$ at a resolution of 35,000 and calibrated with Thermo calibration solution. 
Fig. 1. Representative positive ion mass spectra. a Extracted ion chromatogram at $\mathrm{m} / z$ 371.255-371.260 of LC/MS of pre-dialysis plasma. b Spectrum from peak $c a .16$ min showing the protonated adduct $(\mathrm{m} / \mathrm{z}$ 371) of a compound of mass $370 \mathrm{Da}$ and the loss of a water molecule to $\mathrm{m} / z 353$.

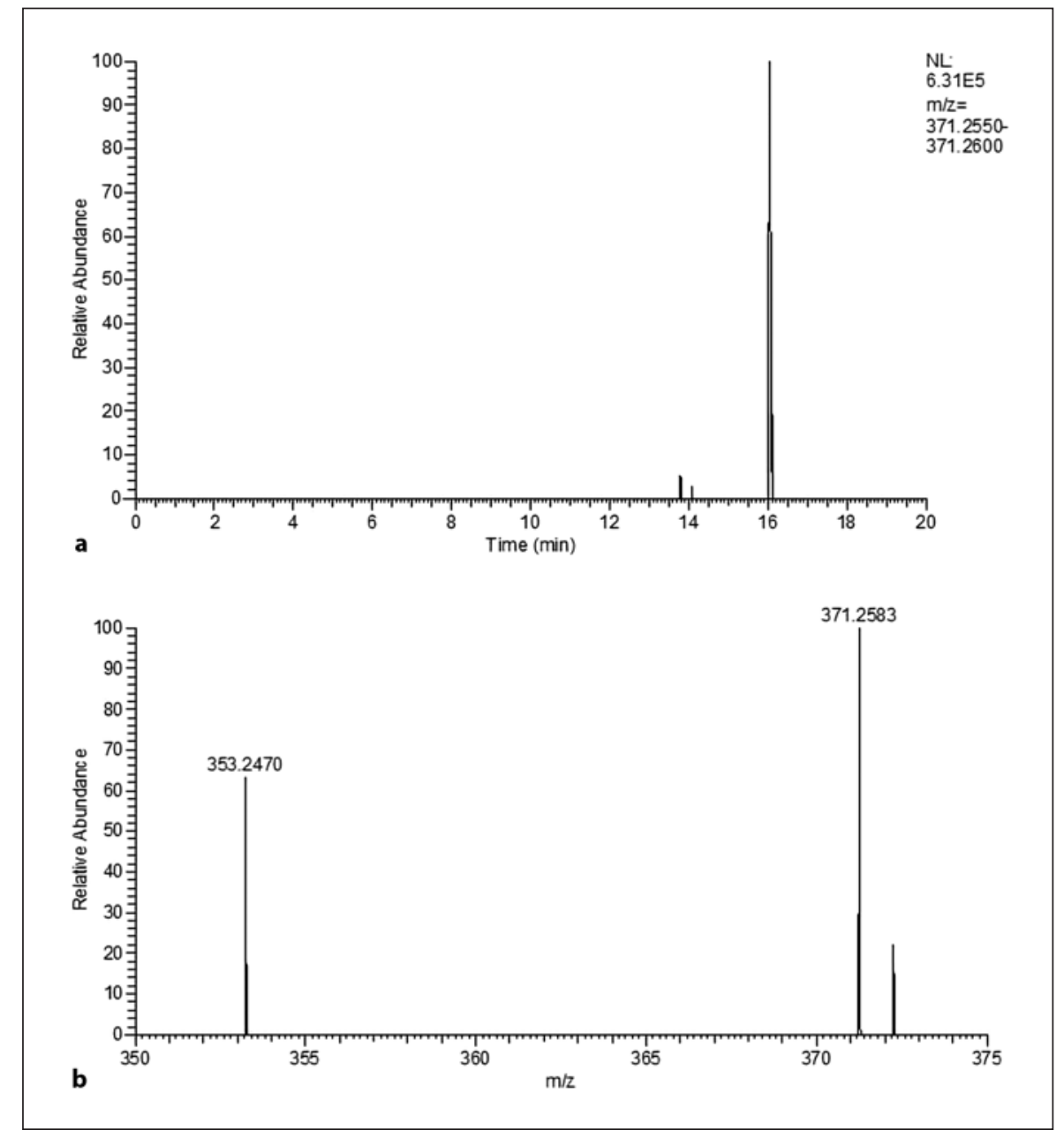

There is no gravimetric standard for the 370-Da molecule as it has never been obtained in weighable quantities. The limit of detection (LOD) for this molecule was therefore expressed solely as the abundance of the protonated adduct (in arbitrary units) at approximately 3 times the background noise. The limit of quantitation (LOQ) was taken to be approximately 10 times the background noise. An authentic signal corresponding to the $370-\mathrm{Da}$ molecule was considered to be present when at a retention time of $\sim 16.0 \mathrm{~min}$, the protonated adduct of the molecule with an accurate mass $(\mathrm{m} / \mathrm{z})$ within $2 \mathrm{ppm}$ of 371.2581 was seen. Under the conditions in this LC/MS system, the material ionised only as its protonated adduct which simplified the quantitation of its abundance. These abundances allow for an assessment of the relative concentration of the material in a sample rather than its absolute value.

Bufalin was obtained from Cayman Chemical Co., Cambridge Bioscience Ltd., Cambridge, UK. The LOD for bufalin was determined by adding a range of bufalin concentrations to control plasma and measuring the abundance of the signal at $\mathrm{m} / z 387.2530$ (the protonated adduct of bufalin). The LOD of bufalin added to control plasma was $3 \mathrm{pmol} / \mathrm{L}$.

Normally distributed data were analysed using the Student $t$ test. Non-parametric data were analysed using the Mann-Whitney $\mathrm{U}$ test. There is no universally accepted method for handling "less than" numeric data, though a common technique is to attribute to them a value of LOD/2 or particularly in logarithmically distributed data, $\mathrm{LOD} / \sqrt{ } 2$. We have not attempted to represent numerically or graphically any data below the LOD, nor have we applied normal statistical analysis to the control and dialysis groups that contained a substantial body of such data.

\section{Results}

There were 39 study subjects, 22 of whom were on HDF and 11 were "non-dialysis normal" controls. Ten (45\%) of the HDF patients were male, the mean age was 60 years (range 42-86), 16 (73\%) were white, 4 (18\%) were black, 1 (4.5\%) was Asian, and 1 (4.5\%) was mixed race. In the control group, $8(72.7 \%)$ were male, the mean age was 55 years (range 42-77), 6 (54.5\%) were white, 4 (36.3\%) were Asian, and 1 (9.2\%) was black.

Nineteen $(86 \%)$ of the HDF patients were on anti-hypertensives: $5(23 \%)$ on angiotensin-converting enzyme 
Fig. 2. Abundance of $m / z 371$ ion. Abundance of the 371 ion in all samples that exceeded the LOQ of 300,000 arbitrary units. In the dialysis patients, the one sample that fell between the LOD and the LOQ has not been plotted, nor have the 14 samples that fell below the LOD. In the control group, 8 samples that fell below the LOD have not been plotted.

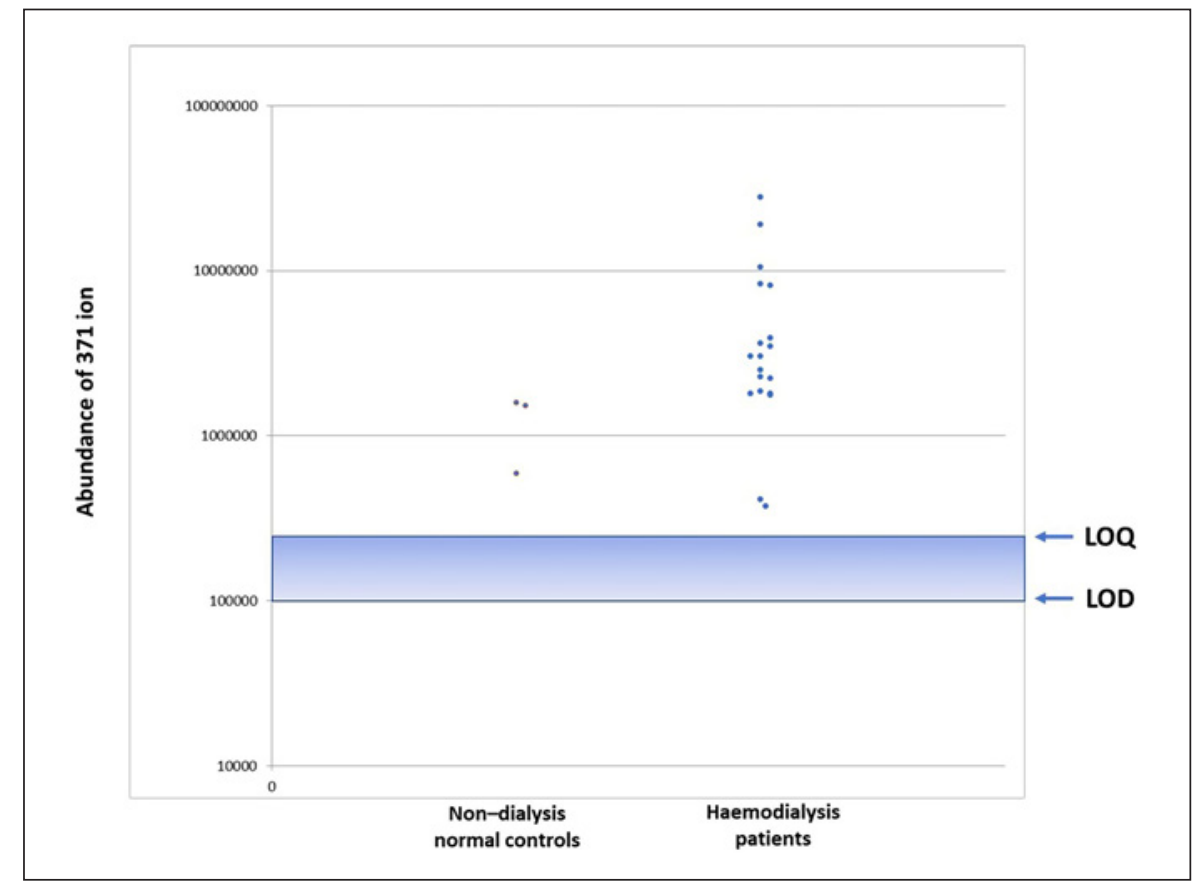

inhibitors, 1 on an angiotensin receptor blocker, and 8 (36\%) on loop diuretics. In the control group, 1 (9.2\%) was on an angiotensin receptor blocker, but none of the others was on anti-hypertensives.

A representative positive ion mass spectrum from a "positive" patient is shown in Figure 1. The accurate mass of the protonated adduct (371.2583) corresponds to the molecular formula $\mathrm{C}_{24} \mathrm{H}_{34} \mathrm{O}_{3}$ with an error of $<2$ ppm and the single water loss (to $\mathrm{m} / \mathrm{z} 353$ ), indicating the presence of one hydroxyl group as in previous studies [8].

In 8 of the 11 controls, the abundance of the 371 ion was below the LOD. No numeric value has been attributed to these samples other than to state that they were all $<100,000$ abundance (arbitrary units). Three control samples exceeded the LOD (and the LOQ) by a small extent (Fig. 2). The median value for the controls was less than LOD.

Sixty-six (33 pre- and 33 post-dialysis) samples were obtained from 22 dialysis patients. In 19 (58\%) of the 33 pre-dialysis plasma samples, the $370-\mathrm{Da}$ molecule was detectable as its protonated adduct $(\mathrm{m} / z$ 371). In one subject, its value fell between the LOD and the LOQ $(300,000$ arbitrary units), and in 13, it was $<$ LOD. Numeric values for the abundance of the 370-Da material were assigned to the samples above the LOQ. The results are shown in Figure 2, but only those samples with an abundance > LOQ have been plotted. In the 33 post-dialysis samples, $23(69 \%)$ were $<$ LOD, one was between the LOD and the LOQ, and $9(27 \%)$ were above the LOQ.

370-Da Inhibitor of the Sodium Pump in Haemodialysis Patients
No significant demographic differences were observed between the population whose pre-dialysis plasma had an abundance of the 370-Da molecule above ("positive") or below ("negative") the LOQ. The average blood pressure was $136 / 70$ in the "negative" group and 157/68 in the "positive" group ( $p>0.2$ for the systolic blood pressure), but interpretation is complicated by the widespread use of anti-hypertensive drugs in both groups. No differences were observed between the types of anti-hypertensive medication used in the 2 groups.

The median pre-dialysis weight in the population with measurable $\mathrm{m} / z 371$ ion abundance was 78.9 (range 43.8$144) \mathrm{kg}$ versus 65.9 (range 45.2-94.6) kg in the "negative" group $(p>0.1)$. No differences were observed in the planned and actual weight loss over a dialysis session in the 2 groups. The data on BMI, medication, demographics, blood pressure, and dialysis parameters are shown in online supplementary Tables 1-3 (for all online suppl. material, see www.karger.com/doi/10.1159/000521202).

\section{Effect of Dialysis}

Thirty-three paired (pre- and post-dialysis) samples were obtained. Thirteen of these pairs had undetectable levels of the 370-Da molecule in both the pre- and postdialysis samples. One pair was between the LOD and the LOQ in both samples.

The remaining 19 pairs had detectable $370-\mathrm{Da}$ molecule in their pre-dialysis plasma, and in the correspond- 
Table 1. Percentage of the 370-Da molecule remaining after dialysis compared with that of urea
Fig. 3. Effect of dialysis on the $m / z 371 \mathrm{~mol}$ ecule. The change in the abundance of the 371 ion after dialysis in pairs where the predialysis sample was $>$ LOQ. The pre-dialysis values have been shown as a common origin, with the post-dialysis samples representing the percentage change from the pre-dialysis value. The samples represented by the dotted lines are from patient 7 , measured on 3 separate occasions. The dashed lines represent patient 10 (see text). Ten pairs have not been included, as the post-value was not numerical, but simply < LOD. It can, however, be calculated that the mean post-value would have been $<13 \%$ of the pre-dialysis sample (see text).
Percentage of compound remaining post-dialysis

370-Da molecule urea

$\begin{array}{lll}\text { Study average (excluding pts } 7 \text { and 10) } & <13 & 33 \\ \text { Patient } 7 \text { (3 incomplete dialysis sessions) }^{1} & 109 \text { (mean of 3) } & 48 \text { (mean of 3) } \\ \text { Patient } 7 \text { (1 completed dialysis session) }{ }^{2} & <12 & 51 \\ \text { Patient } 10 & 88 & 26\end{array}$

${ }^{1}$ Three dialysis sessions during which the planned ultrafiltration could not be effected (see text). ${ }^{2}$ The findings in 1 uncomplicated dialysis session.

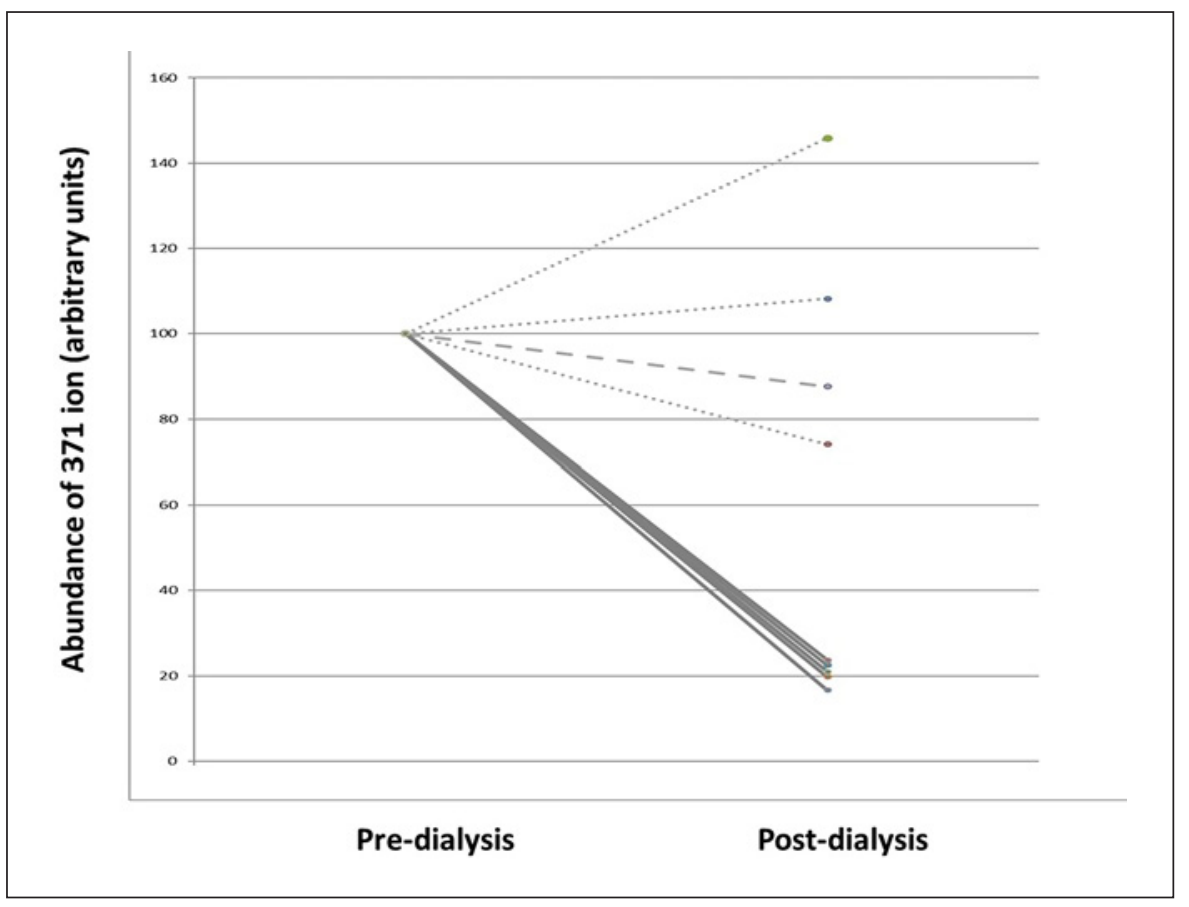

ing post-dialysis samples, 17 exhibited a marked fall in the abundance of the $m / z 371$ ion across the dialysis session. Ten were below the LOD, but 7 had measurable levels above the LOQ, and in these samples, the fall in abundance of the 370-Da molecule was to an average value of $22 \%$ of the pre-dialysis value. Attributing a value of " $<$ LOD" to the 10 unplotted post-dialysis samples means that the average fall for this group was $<13 \%$ of the predialysis value. The corresponding fall for urea, molecular weight (MW) 60, was to 33\% and for creatinine (MW 113 ) was to $38 \%$ of their pre-dialysis values. Thus, the reduction in the abundance of the $370-\mathrm{Da}$ molecule was substantially greater than that of urea despite its molecular weight being over 6 times greater (Table 1).
Two individuals exhibited no significant fall in the abundance of the 370-Da molecule, and each was atypical in terms of their fluid status. In one of these patients (patient 7), it was possible to observe pre- and post-dialysis samples on 4 separate occasions. This patient was morbidly obese $(\sim 140 \mathrm{~kg})$ to an extent that precluded regular weighing either pre- or post-dialysis and was clinically chronically volume overloaded. However, attempts to remove fluid during dialysis usually resulted in haemodynamic instability that terminated any further fluid removal and sometimes required a curtailing of the dialysis session along with a limited infusion of isotonic saline to stabilize the situation. On 3 separate occasions, where this occurred, the post-dialysis 370-Da molecule's abundance 
Fig. 4. Extracted ion chromatograms - patient 10. Extracted ion chromatogram at $m / z$ 371.2355-371.2360 for LC/MS of predialysis and post-dialysis plasma in patient 10. Average accurate masses at peak apex are displayed alongside peaks.

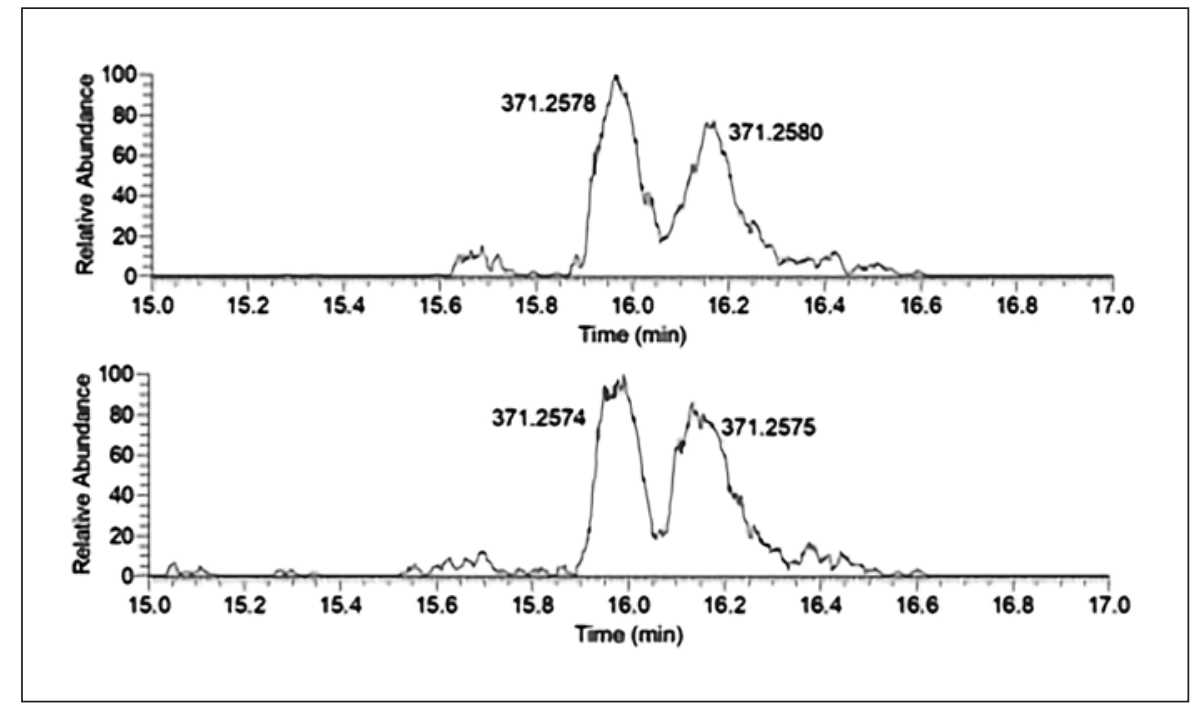

either fell only minimally, or actually increased across the dialysis session. However, on a fourth dialysis session, an uncomplicated treatment was completed, and $2.5 \mathrm{~L}$ of fluid was successfully removed. The weight loss across the dialysis was not measured however for reasons described above. On that occasion, the post-dialysis sample showed that the abundance of the 370-Da molecule had fallen to less than the LOD, representing a value of less than $12 \%$ of the pre-dialysis abundance.

The second patient (patient 10) was also atypical, with a native urine output of approximately $2 \mathrm{~L} /$ day, often requiring no ultrafiltration on dialysis, was normotensive on no anti-hypertensive medication, and remained well on two 3-h dialysis sessions a week, against an average of $3 \times 4$ h per week for the other individuals studied. However, he had previously intermittently shown signs of volume overload. On the day the samples were taken, his pre-dialysis weight was $64.7 \mathrm{~kg}$, but his target weight was $63 \mathrm{~kg} .0 .8 \mathrm{~L}$ of fluid was removed during the session, and his post-dialysis weight was $64.2 \mathrm{~kg}$, suggesting that he remained fluid overloaded post-dialysis, and the abundance of the 370 Da molecule fell by only $12 \%$ across the dialysis session. These results are shown in Figure 3. Both patients 7 and 10 had lower than average dialysis times, and their changes in blood urea and the 371 ion are documented in Table 1 alongside the average values for other patients in the study.

\section{Evidence of Isomerism of the 370-Da Molecule}

The 370-Da molecule was almost invariably seen as a single peak at $\mathrm{m} / \mathrm{z} 371$ (the protonated adduct) on all occasions where the LOD was exceeded. However, in pa- tient 10, the extracted ion chromatogram of the LC/MS showed 2 molecular species incompletely resolved by the HPLC column with an identical accurate mass of 371.2581 (within $2 \mathrm{ppm}$ ) and hence a common molecular (elemental) formula (Fig. 4). This phenomenon was also seen in patient 7 , though in that case, the second peak was of lower abundance than the first.

\section{Discussion}

The demonstration that the sodium pump of leucocytes was inhibited in patients with untreated hypertension and the subsequent observation that this effect was transmittable by incubation in plasma has parallels with similar studies in uraemia and volume expansion (the natriuretic hormone). The findings were used to generate a hypothesis whereby such pump inhibition (assumed to be a manifestation of salt handling) led to an increase in arteriolar tone and a raised systemic vascular resistance [14]. The demonstration that foetal cord plasma contains a substance that cross-reacts with digoxin antibodies and inhibits the sodium pump [15] naturally leads to questions as to whether this reflects the necessary process in the foetus to maintain salt and water balance in utero.

We previously reported the extraction of an inhibitor of the sodium pump of human leucocytes from human placentas by way of dialysis, entrapment of the less polar compounds on C18 SPE cartridges, and subsequent reversed-phase high-performance liquid chromatography [8]. In 1 patient, who was undergoing repeated plasma exchange for cryoglobulinaemia, we were able to study 
exchanged plasma in both the normal and volume-expanded state when 2 sessions of plasma exchange were interspersed with an acute nephritic event with haematuria, proteinuria, fluid retention, and a fall in glomerular filtration rate. The previous lack of any measurable effect on the leucocyte sodium pump was replaced by significant inhibition, and in the corresponding sample, the 370-Da material became detectable in the plasma [9]. In subsequent studies involving ultrafiltrate or exchanged plasma from volume-expanded patients, we identified a molecule indistinguishable from the placental material in terms of its molecular formula and its fragmentation pattern in negative ion MS and which was again associated with inhibition of the sodium pump [10].

The performance of each of these mass spectrometric techniques described above was much lower than is achievable with modern instrumentation where not only the sensitivity but also the resolution of the process has been radically enhanced. These improvements induced us to look at plasma of patients receiving regular haemodialysis, for what were likely to be much lower levels of the 370-Da material - concentrations at which classical sodium transport studies would be impractical.

In the current study, the abundance of the $370-\mathrm{Da}$ molecule in pre-dialysis plasma samples showed a high degree of variability. Thirteen samples had values indistinguishable from the normotensive, biochemically normal controls, demonstrating that end-stage renal failure requiring dialysis does not in itself necessarily result in an abundance of the 370-Da molecule in plasma at greater than control values. Only 1 sample fell between the LOD and the LOQ. In the other 19 samples, values ranged from just exceeding to over 30 times the LOQ. If the LOD of the 370-Da molecule was to be comparable to that of bufalin, this maximum would represent a value of approximately $100 \mathrm{pmol} / \mathrm{L}$. This is a high range for a presumed endogenous substance, but is comparable to values seen for vasopressin [16] and brain-type natriuretic peptide [17].

\section{Effect of Dialysis}

The principal physical chemical force in the dialyser is diffusion, where molecular size is the most important driver of its removal, but HDF includes a component of ultrafiltration which alters this simple relationship in that all molecules up to and beyond $370 \mathrm{Da}$ have effectively identical clearances by this route. However, this is designed to be a small component of the HDF process and does not have a major disturbing effect on the relationship between molecular size and clearance.
Other factors could also be expected to complicate this relationship, in particular the apparent volume of distribution of the molecule under study, where binding to and slow dissociation from the sodium pump would be especially relevant. However, the fact that in 2 patients, the abundance of the 370-Da molecule in plasma could largely be maintained, or even increased, across a dialysis session (seen in 4 samples from 2 patients) tends to refute the idea that clearance via the dialyser can be considered the sole, or even the most important cause for the fall across dialysis in the remaining patients.

In the majority of patients who had a quantifiable level of the $370-\mathrm{Da}$ molecule in pre-dialysis samples, the post-dialysis level fell disproportionately to its molecular weight, which also suggests that rather than attributing this fall to clearance in the dialyser, we should be considering that dialysis results in physiological changes that either curtail the endogenous production of the material or enhance its catabolism. Support for the view that clearance by the dialyser is not necessarily a major influence on the post-dialysis abundance of the molecule is provided by patients 7 and 10, who largely maintained or even increased the abundance of the 370-Da molecule across the dialysis session. There is no indication from the data in Table 1 that the reduced dialysis time seen in patient 10 was influential in altering the ratio of urea to the 370 -Da molecule's removal, and while patient 7 , as expected, clearly had an impaired urea clearance in each of the 4 treatment sessions studied, it seems unreasonable to attribute the divergence of the changes in urea and the 370-Da molecule's abundance to restricted removal of the latter by the dialyser, particularly when reference is made to the one dialysis session where the planned fluid removal was able to be accomplished.

\section{Isomers}

The observation that the extracted ion chromatogram twice (patients 7 and 10) showed reduplication of the $\mathrm{m} / \mathrm{z}$ 371 ion with partial resolution of the peaks and that these had identical accurate masses provides convincing evidence of isomerism of this molecule. This invites comparison with a previous report involving 5 volume-expanded patients with renal impairment, all of whom generated a sodium pump inhibitor in their plasma and in whom the corresponding HPLC fraction showed the presence of an ion of $\mathrm{m} / z 371$ (protonated $370 \mathrm{Da}$ ) on positive ion MS. In those earlier studies, the chromatographed material was collected as 2-min fractions to allow for both mass spectrometric analysis and the determination of inhibition of the sodium pump. This led to seeing the spec- 
tra of the 2 possible isomers in 1 or 2 sample tubes, rather than with the temporal separation observed here.

Four of the 5 patients in that study showed a complex pattern on negative ion MS in which the corresponding deprotonated ion $(\mathrm{m} / z$ 369) fragmented ultimately to either $\mathrm{m} / z 97$ and 80 or $m / z 113$ and 69 [9]. It seems likely that these 2 processes represent different manifestations of the convincing isomerism demonstrated in patients 7 and 10 . That being the case, the absence of evidence of isomerism in the majority of the patients studied here raises the possibility that the time course of volume expansion may be an important factor in generating the second isomer. Given that different isoforms of the sodium pump are known to exist in general in human cells, it is possible that further characterization of these isomers might demonstrate a degree of specificity for different pump isoforms.

\section{Limitations and Implications}

As a consequence of never having more than nanogram quantities of the 370-Da molecule, we were unable to convert our abundance data into a meaningful concentration, although the LOD of bufalin of $3 \mathrm{pmol} / \mathrm{L}$ (a molecule whose molecular formula differs from the $370-\mathrm{Da}$ molecule by a single oxygen atom, in the form of a hydroxyl group) makes it possible to infer approximate limits to the range of abundances seen in the plasma of these uraemic patients.

\section{Conclusion}

Our study presents interesting data concerning the abundance of the 370-Da molecule in advanced chronic kidney disease and its rapid change in response to haemodialysis in most patients. Our previous studies relating to the characterization of an inhibitor of the sodium pump in placental extracts and its subsequent demonstration in volume-expanded individuals involved approximately 1,000 -fold concentration of the source material, required by the sensitivity of the mass spectrometers of the time. The subsequent increased sensitivity (and resolution) of mass spectrometers led us to postulate that we would be able to detect the molecule in a "clinical" sized sample of plasma in susceptible individuals. This proved to be the case in that we could detect the molecule in a vacutainer sample of plasma in approximately half of the patients immediately prior to a haemodialysis session. This implies that this technique could usefully be applied to other populations such as hypertension [18], pregnancy [15], and the neonatal state [19], where either the sodium pump is known to be inhibited, or their plasma is known to contain substances that cross-react with digoxin antibodies.

\section{Acknowledgments}

The authors are grateful to Drs. Mark Dockrell, Rebecca Suckling, and Pauline Swift (St. Helier Hospital) for their help during this study and to Professor Timothy Gant (Public Health England), Dr. Juan Mason (Portsmouth, UK), and Professor Gary Nicholls (Christchurch, New Zealand) for their helpful comments during the preparation of this manuscript.

\section{Statement of Ethics}

The study was approved by the London-Bloomsbury Research Ethics Committee and the United Kingdom Health Research Authority (Ethics Approval No. 16/LO/0364). Written informed consent was obtained from all patients in this study.

\section{Conflict of Interest Statement}

The authors have no personal or financial conflicts of interests in respect of this study.

\section{Funding Sources}

The work was partly supported by the South West Thames Institute for Renal Research.

\section{Author Contributions}

Dr. Hilton was responsible for the overall conduct of the research, Dr. Makanjuola for its clinical aspects, and Dr. Lord for the mass spectrometric analysis. All individuals acknowledged have seen and approved the mention of their name in this paper.

\section{Data Availability Statement}

The source data can be found in online supplementary Tables $1-3$.

References

1 Skou JC. The influence of some cations on an adenosine triphosphatase from peripheral nerves. Biochim Biophys Acta. 1957 Feb; 23(2):394-401.

2 de Wardener HE, Mills IH, Clapham WF, Hayter CJ. Studies on the efferent mechanism of the sodium diuresis which follows the administration of intravenous saline in the dog. Clin Sci. 1961;21:249-58. 
3 Cole CH, Balfe JW, Welt LG. Induction of a ouabain-sensitive ATPase defect by uremic plasma. Trans Assoc Am Physicians. 1968;81: 213-20.

4 Edmondson RP, Thomas RD, Hilton PJ, Patrick J, Jones NF. Abnormal leucocyte composition and sodium transport in essential hypertension. Lancet. 1975;1:1003-5.

5 Gray HH, Hilton PJ, Richardson PJ. Effect of serum from patients with essential hypertension on sodium transport in normal leucocytes. Clin Sci 1986;70:583-6.

6 Mathews WR, DuCharme DW, Hamlyn JM, Harris DW, Mandel F, Clark MA, et al. Mass spectral characterization of an endogenous digitalis-like factor from human plasma for structural analysis. Hypertension. 1991;17: 923-9.

7 Bagrov AY, Dmetrieva RI, Fedorova OV, Kazakov GP, Roukoyatkina NI, Shpen VM. Endogenous marinobufagenin-like immunoreactive substance. A possible $\mathrm{Na}$, K-ATPase inhibitor with vasoconstrictive activity. Am J Hypertension. 1996;9(10 Pt 1):982-90.
8 Hilton PJ, White RW, Lord GA, Garner GV, Gordon DB, Hilton MJ, et al. An inhibitor of the sodium pump obtained from human placenta. Lancet. 1996;348:303-5.

9 McKinnon W, Lord GA, Forni LG, Hilton PJ. Circulating sodium pump inhibitors in five volume-expanded humans. J Hypertens. 2003;21:2315-21.

10 Wright DA, Lord GA, White RW, Hilton MJ, Gordon DB, Hilton PJ. Evidence for circulating bufenolide in a volume expanded human. Lancet. 1997;350:409.

11 Kind T, Fiehn O. Advances in structure elucidation of small molecules using mass spectrometry. Bioanal Rev. 2010;2:23-60.

12 Fenn JB. Electrospray: wings for molecular elephants (Nobel Lecture). Angew Chem Int Ed. 2003;42:3871-94.

13 Gross ML. Accurate masses for structure confirmation. J Am Soc Mass Spectrom. 1994;5: 57.

14 de Wardener HE, MacGregor GA. Dahl's hypothesis that a saluretic substance may be responsible for a sustained rise in arterial pressure: its possible role in essential hypertension. Kidney Int. 1980;18:1-9.
15 Graves SW, Valdes R, Brown BA, Knight AB, Craig HR. Endogenous digoxin-immunoreactive substance in human pregnancies. Am J Obst Gynecol. 1984;150:83-5.

16 Weitzman RE, Glatz TH, Fisher DA. The effect of hemorrhage and hypertonic saline upon plasma oxytocin and arginine vasopressin in conscious dogs. Endocrinology. 1978; 103:2154-60.

17 Murdoch DR, Byrne J, Morton JJ, McDonagh TA, Robb SD, Clements S, et al. Brain natriuretic peptide is stable in whole blood and can be measured using a simple rapid assay: implications for clinical practice. Heart. 1997;78: 594-7.

18 Hilton PJ. Cellular sodium transport in essential hypertension. N Engl J Med. 1986;314: 222-9.

19 Morris JF, McEachern MD, Poston L, Smith SE, Mulvany MJ, Hilton PJ. Evidence for an inhibitor of leucocyte sodium transport in the serum of neonates. Clin Sci. 1987;73:291-7. 